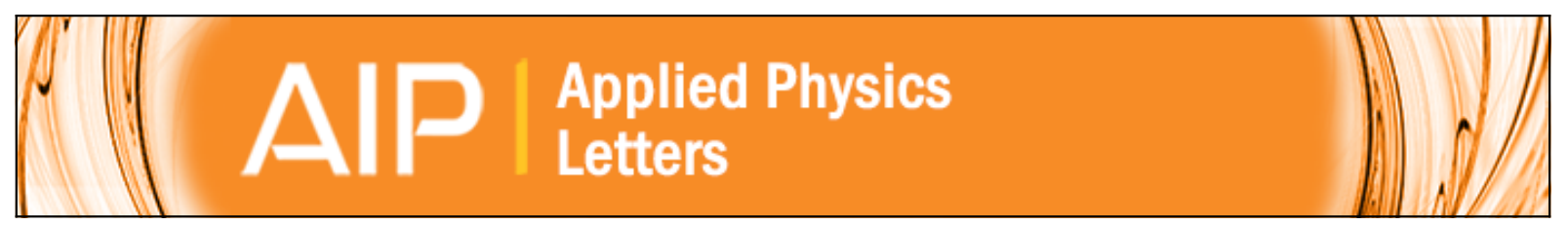

\title{
Temporal dynamics of all-optical switching in quadratic nonlinear directional couplers
}

R. Schiek, A. S. Solntsev, and D. N. Neshev

Citation: Applied Physics Letters 100, 111117 (2012); doi: 10.1063/1.3696030

View online: http://dx.doi.org/10.1063/1.3696030

View Table of Contents: http://scitation.aip.org/content/aip/journal/apl/100/11?ver=pdfcov

Published by the AIP Publishing

\section{Articles you may be interested in}

All-optical switching in optically induced nonlinear waveguide couplers

Appl. Phys. Lett. 104, 261111 (2014); 10.1063/1.4886414

All-optical polarization switch in a quadratic nonlinear photonic quasicrystal

Appl. Phys. Lett. 94, 091108 (2009); 10.1063/1.3090488

Switchable Al x Ga 1 - x As all-optical delay line at $1.55 \mu \mathrm{m}$

Appl. Phys. Lett. 90, 101112 (2007); 10.1063/1.2679838

Pulse-width selective all-optical switching

Appl. Phys. Lett. 88, 181110 (2006); 10.1063/1.2200755

Soliton switching in three-core nonlinear directional fiber couplers

J. Appl. Phys. 84, 1834 (1998); 10.1063/1.368615

\section{AIP $\left.\right|_{\text {APL Photonics }}$}

APL Photonics is pleased to announce Benjamin Eggleton as its Editor-in-Chief

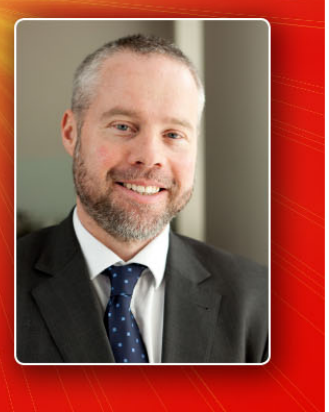




\title{
Temporal dynamics of all-optical switching in quadratic nonlinear directional couplers
}

\author{
R. Schiek, ${ }^{1, a)}$ A. S. Solntsev, ${ }^{2}$ and D. N. Neshev ${ }^{2}$ \\ ${ }^{1}$ Electrical Engineering, University of Applied Sciences Regensburg, Prüfeninger Strasse 58, \\ 93049 Regensburg, Germany \\ ${ }^{2}$ Nonlinear Physics Centre, Centre for Ultrahigh-Bandwidth Devices for Optical Systems (CUDOS), \\ Research School of Physics and Engineering, Australian National University, Canberra ACT 0200, Australia
}

(Received 20 February 2012; accepted 29 February 2012; published online 16 March 2012)

\begin{abstract}
We study the temporal dynamics of all-optical switching in nonlinear directional couplers in periodically poled lithium niobate. The characteristic features of such switching, including asymmetric pulse break-up and back-switching were measured in full agreement with the theoretical predictions. Based on the time-resolved measurement of intensity-dependent switching, finally the theoretically long-known continuous-wave switching curve has experimentally been confirmed. (C) 2012 American Institute of Physics. [http://dx.doi.org/10.1063/1.3696030]
\end{abstract}

All-optical switching in nonlinear directional couplers (NLDCs) is one of the most fundamental processes for studies of intensity-dependent light propagation. Importantly, the intensity-dependent mode coupling in the NLDC forms the basis for an understanding of wave propagation in other more complex nonlinear systems like waveguide arrays and solitons in slab waveguides. The propagation constants of the modes of a system are changed by the nonlinearity. The resulting modification of the coupling between the modes and their superposition finally determines the intensity pattern at the output of the system dependent on the input power. For ultrafast all-optical switching, usually short pulses and fast nonlinear processes such as Kerr-like or cascaded quadratic nonlinearities are employed. The quadratic NLDC is far superior in terms of power requirements and flexibility; however, due to the involvement of more than one optical frequency and a strong frequency dependence of the nonlinearity, the system becomes more complex.

Theoretically the NLDC has been understood very well for 30 years ${ }^{1}$ and NLDCs based on cubic Kerr-type nonlinearities have intensively been studied in the laboratories. ${ }^{2-5}$ Following these works, quadratic NLDCs were also studied theoretically ${ }^{6}$ and experimentally, ${ }^{7,8}$ revealing their greater flexibility due to a tunability of the cascaded quadratic nonlinearity. Similar to the cubic case, it was shown that complete switching is in general prevented by pulse break-up for most of the experimentally available pulses. ${ }^{6,9}$

While there have been a number of experiments aiming to directly observe the temporal dynamics of short pulses in cubic all-optical switches, ${ }^{3,10}$ a measurement of the pulse shaping in a quadratic NLDC was lacking. Due to the complexity of the pulse dynamics, determined by the frequency dependence of nonlinearity and group velocity, it is interesting to directly measure the performance of such quadratic NLDCs and to compare it with the existing theoretical studies. ${ }^{6}$ In this letter, we present the results of high-resolution measurements of the temporal pulse shape of the output from quadratic nonlinear couplers with a frequency-resolved

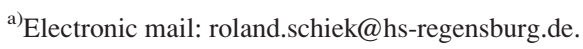

optical gating technology (FROG). We observed and elucidated the details of temporal switching, including incomplete switching in the pulse wings, back-switching in the center, and an asymmetry of the resulting pulse break-up.

Both, single channel waveguides and pairs of linearly coupled waveguides were fabricated by indiffusion of $7-\mu \mathrm{m}-$ wide and 100 -nm-thick titanium stripes at $1060^{\circ} \mathrm{C}$ for $8.5 \mathrm{~h}$ in a $L=50$-mm-long $Z$-cut congruent lithium niobate $\left(\mathrm{LiNbO}_{3}\right)$ crystal with propagation along the crystallographic $\mathrm{X}$ axis. To achieve large intensity-dependent phase shifts for the fundamental wave (FW) due to the cascaded quadratic nonlinearity, type I wavelength- and temperature-tunable quasi-phase-matched (QPM) second-harmonic generation (SHG) was implemented. An electric-field-poled QPM grating with a period of $\Lambda=16.751 \mu \mathrm{m}$ yielded phase-matching for SHG between the $\mathrm{TM}_{00}$ modes for a FW wavelength of $\lambda_{\mathrm{PM}}=1522 \mathrm{~nm}$ at room temperature. The end facets were polished for end-fire coupling and were anti-reflection coated, having reflectivities of $\approx 1 \%$ for the $\mathrm{FW}$ and $14 \%$ for the second harmonic ( $\mathrm{SH})$. Linear losses between 0.2 and $0.23 \frac{\mathrm{dB}}{\mathrm{cm}}$ for the FW and 0.65 and $0.75 \frac{\mathrm{dB}}{\mathrm{cm}}$ for the $\mathrm{SH}$ have been measured. To prevent a photorefractive (PR) detuning of the SHG resonance and to minimize a PR shift of the coupling length, the sample was heated to $170-240{ }^{\circ} \mathrm{C}$ which increased $\lambda_{\mathrm{PM}}$ close to $1550 \mathrm{~nm}$.

The theoretical description of the switching dynamics is based on a coupled-mode theory in the frequency domain ${ }^{6}$ using the measured input pulse envelope and phase. The parameters for the model were all measured carefully and the modeling yields excellent quantitative agreement to the measurements without any fitting parameters. At a wavelength of $1550 \mathrm{~nm}$, a coupler with a center-to-center waveguide separation of $18.3 \mu \mathrm{m}$ with a coupling length of $L_{\mathrm{c}}=54 \mathrm{~mm}$ is the closest to a half-beat-length coupler in our sample. The $\mathrm{SH}$ modes of both arms are not coupled due to the strong confinement of these modes. The propagation constants $\beta_{\mathrm{FW}}$ and $\beta_{\mathrm{SH}}$ were calculated with a mode solver. The overlap integral $K^{(2)}=\iint \mathrm{d} x \mathrm{~d} y e_{\mathrm{FW}}^{2}(x, y) e_{\mathrm{SH}}^{*}(x, y)$ $=8.3 \cdot 10^{8} \frac{\mathrm{V}^{3}}{\mathrm{~m}}$ for SHG was determined from mode-shape measurements. Here, $e_{\mathrm{FW} / \mathrm{SH}}(x, y)$ are the transverse electric fields of the waveguide modes normalized to $1 \mathrm{~W}$. The 
quadratic nonlinear susceptibility in our system was measured to be $d_{33}=20.6 \frac{\mathrm{pm}}{\mathrm{V}} \cdot{ }^{11}$

Pulses from an amplified mode-locked fiber laser from Pritel with a repetition rate of $5 \mathrm{MHz}$, at a wavelength of $\lambda_{\mathrm{FW}}=1550.88 \mathrm{~nm}$, bandwidth of $0.35 \mathrm{~nm}$, and a pulse length of $9 \mathrm{ps}$ at full width at half maximum were end-fire coupled into the waveguides. The asymmetric SHG tuning curve from a single waveguide in Fig. 1(a) indicates a nonuniform distribution of the wave-vector mismatch due to a temperature gradient in the heated crystal. ${ }^{12}$ The SH output is minimized for a positive wave-vector mismatch $\Delta k=2 \beta_{\mathrm{FW}}-\beta_{\mathrm{SH}}+\frac{2 \pi}{\Lambda}$ below the phase-matching temperature $T_{\mathrm{PM}}=219^{\circ} \mathrm{C}$. The temperature dependence of the normalized wave-vector mismatch is given by $\Delta k L=1.8 \pi \times\left(219-T /{ }^{\circ} \mathrm{C}\right)$. The total throughput of $80 \%$ is reduced close to phase-matching when the FW is depleted and part of the energy propagates in the more lossy SH mode.

All-optical switching in the NLDC was investigated by coupling the light into one arm of a half-beat-length coupler and observing the output dependent on the input power. The FW and SH outputs from both coupler arms were separated spatially and imaged onto InGaAs and CCD cameras. The FW and SH average power from both arms was measured with four photodiodes. By using the measured input pulse shape [see also Fig. 2(d)] and the pulse repetition rate, the average FW power is converted to the peak power $P_{\text {in }}$ and to the pulse energy $E$. Fig. 1(b) shows a typical measured switching curve showing the output pulse energy $E_{\text {out }}$ from the cross
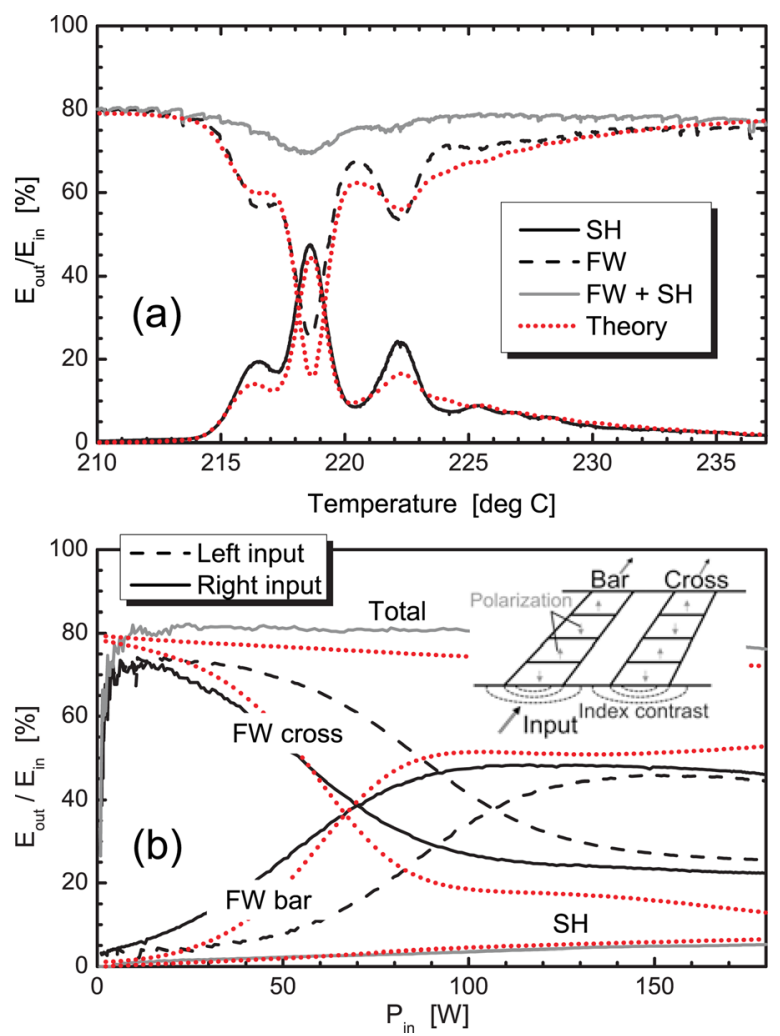

FIG. 1. (Color online) (a) Temperature-tuned SHG and FW-depletion from a single waveguide for an input peak power $P_{\text {in }}=1.6 \mathrm{~W}$. (b) Normalized FW output pulse energy from the bar and the cross arm (see inset) versus $P_{\text {in }}$ from a half-beat-length coupler for left- (dashed line) and right-arm (solid line) input; $L_{\mathrm{c}}=54 \mathrm{~mm}, 210^{\circ} \mathrm{C}$. The $\mathrm{SH}$ and the total throughput are also shown. Dotted lines show theory.
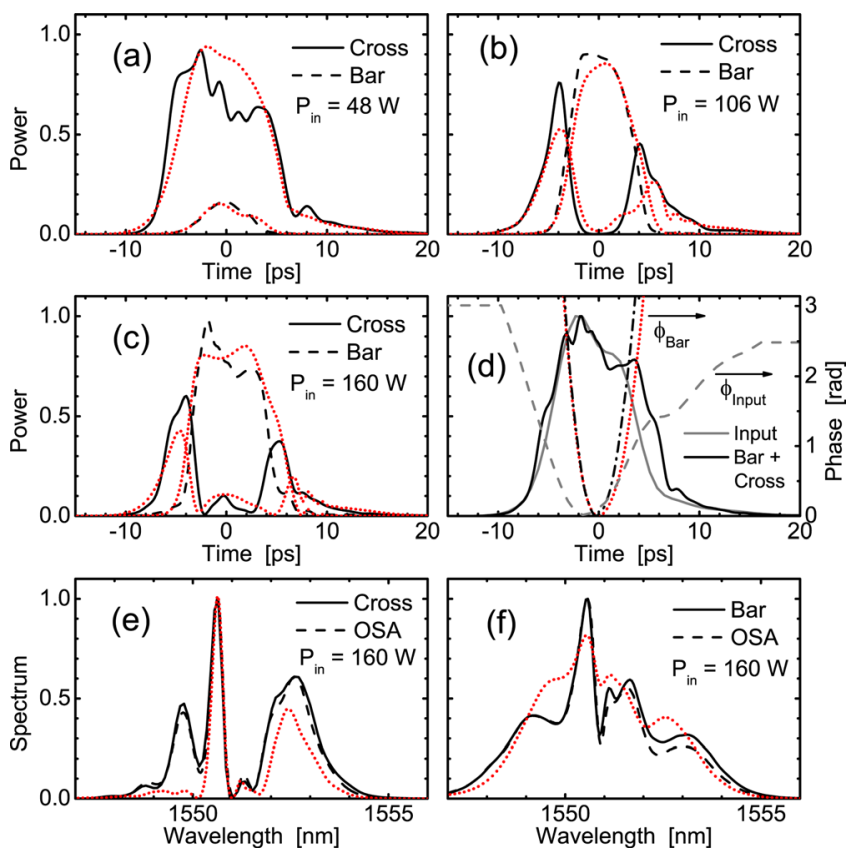

FIG. 2. (Color online) Normalized pulse traces from the output arms of a half-beat-length coupler; $L_{\mathrm{c}}=54 \mathrm{~mm}$. (a) $P_{\text {in }}=48 \mathrm{~W}<P_{\mathrm{sa}}$, (b) $P_{\text {in }}=106 \mathrm{~W}$ $\approx 1.2 \times P_{\text {sa }}$, (c) $P_{\text {in }}=160 \mathrm{~W}>P_{\text {sa. }}$. (d) Input pulse compared to the total FW output; $P_{\text {in }}=160 \mathrm{~W}$. The phases of the input pulse and the output pulse from the bar arm are also shown. (e) and (f) Measured and recovered spectra (from FROG traces) of the pulses from the cross and the bar arm in (c). The temperature was $205^{\circ} \mathrm{C}$. Dotted lines show theory.

and the bar arm normalized to the input pulse energy $E_{\text {in }}$. The switching power $P_{\mathrm{sa}}$ is the input peak power for equal average output from both arms. Due to a small asymmetry of both coupler branches, $P_{\mathrm{sa}}$ is not equal for the left- (dashed line) and the right-arm (solid line) input. Furthermore, a small SHinduced PR index change slightly increases this asymmetry for larger powers. In theory (dotted curves in Fig. 1), we neglect the asymmetry which explains the deviations between calculated and measured data. Operating at temperatures below phase-matching, the generated SH power is small [see Fig. 1(a)] and the switching does not suffer from FW depletion. The total throughput in Fig. 1(b) is therefore relatively constant. Only for larger input powers when most of the FW and the generated SH stay in the bar arm, larger SH losses yield an observable reduced total throughput.

The power-dependent switching was characterized at different temperatures and in different couplers. The dependence of $P_{\mathrm{sa}}$ on the phase-mismatch and on the coupling length agrees well with the theory for all measurements. Importantly, the couplers switch also at higher temperatures with negative phase shifts, but because of stronger SHG, the switching curves are not as clean as with the positive phase shifts.

For a characterization of the temporal dynamics, the FW output pulses of both arms were separately measured with a FROG apparatus from Southern Photonics. The spectra were monitored with an optical spectrum analyzer (OSA). Fig. 2 shows typical switching situations.

At low input power, Fig. 2(a) shows the beginning of switching in the pulse center where the power from the cross arm starts to switch to the bar arm. For larger $P_{\text {in }}$, the strongly increased power in the pulse center in the bar arm coincides with a corresponding, depleted power in the cross 
arm. In the low-intensity parts of the pulse (pulse wings), the light couples in a linear fashion to the cross arm, while the higher-intensity pulse center is switched to the bar arm. The resulting pulse break-up in the cross arm is observed in Figs. 2(b) and 2(c). For $P_{\text {in }}$ slightly above $P_{\text {sa }}$ [Fig. 2(b)], the power in the pulse center equals the power for complete switching and, therefore, the center of the pulse remains in the bar arm. A further increase of $P_{\text {in }}$ [Fig. 2(c)] shows a complete switching of the pulse intensity for two points on both sides of the pulse peak. In the pulse center, back switching is observed as theoretically predicted more than 30 years ago. ${ }^{1}$

The phase of the input pulse shows a slight up-chirp [Fig. 2(d)]. The positive nonlinear phase shift increases this chirp for an increasing power. The resulting larger chirp of the output pulse is shown for the bar arm pulse [Fig. 2(d), dashed-dotted line]. The time direction in the FROG retrieval was found from this increased up-chirp.

Figs. 2(e) and 2(f) show as representative examples the good agreement between retrieved and measured spectra with strong self-phase modulation.

The sum of the powers of both output arms equals relatively well the measured input pulse profile for all the measurements with less than $10 \%$ generated $\mathrm{SH}$, as shown in Fig. 2(d). This shows that in a first approximation, the quadratic cascaded nonlinearity acts quasi-instantaneous for an operation with low FW-depletion and 9-ps-long pulses. However, the observed asymmetry of the switching cannot entirely be explained by the asymmetric laser pulse. Numerical simulations show that the wavelength dependence of the phasemismatch yields different effective nonlinearities for the different spectral components which introduce a kind of nonlinear dispersion. Together with the linear dispersion ( $\mathrm{SH}$ walk-off), the pulse-form-induced asymmetry is magnified and the pulses broaden and develop a tail. Therefore, for the 9-ps-long pulses, the "temporal non-locality" of the cascaded nonlinearity ${ }^{13}$ influences already the pulse propagation and-especially in the second half of the pulses-the assumption of an instantaneous nonlinearity cannot exactly explain the details of the pulse dynamics.

An important consequence of the exact time-resolved measurement of the optical switching is the possibility to determine the continuous-wave (cw) switching curve, too. For a pure instantaneous nonlinearity in a dispersion-free system, it is possible to retrieve the $\mathrm{cw}$ switching curve by plotting the instantaneous normalized powers from both output arms versus the instantaneous input power of the bellshaped input pulse. Corresponding plots of the experimental data indeed show the behavior of switching. However, the deviations of the system from dispersion-free with partly non-instantaneous nonlinearity are strong enough to disturb a so determined $\mathrm{cw}$ switching characteristic in the second half of the pulse. Therefore, in order to measure a cw switching curve as close as possible, traces of switched pulses from both arms were measured with increasing the input power by small increments with the results illustrated in Fig. 3. Determining the ratio of the pulse energies from both channels from the measured switching curve in Fig. 3(c), the pulse traces were calibrated and normalized to the input. The color-coded contour plots in Figs. 3(a) and 3(b) show the calibrated and normalized pulse traces dependent on the
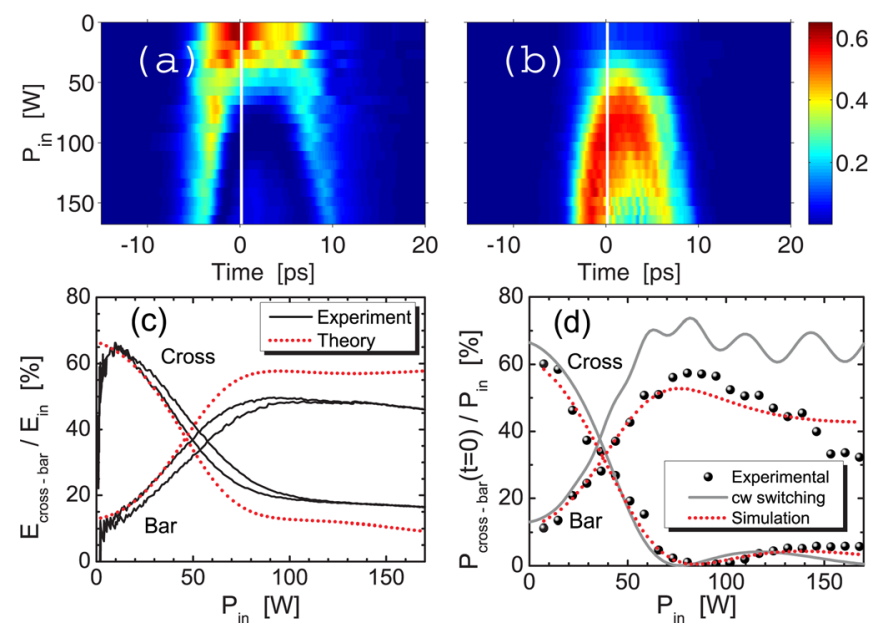

FIG. 3. (Color online) Normalized experimental pulse traces dependent on the input peak power $P_{\text {in }}$ from (a) the cross and (b) the bar arm. A center-tocenter waveguide separation of $18.7 \mu \mathrm{m}$ corresponds to $L_{\mathrm{c}}=68 \mathrm{~mm} ; 210^{\circ} \mathrm{C}$. (c) Measured and calculated switching curve for pulse energies. (d) From (a) and (b) in the pulse center derived switching curve compared to a cw model and a numerical simulation.

input peak power $P_{\text {in }}$. Evaluating only the powers in the pulse center, the switching curve for the momentary peak power shown in Fig. 3(d) was derived. The power scans are marked by white lines in Figs. 3(a) and 3(b). A simulation of the experiment agrees perfectly to the measured data. A scan from calculated contour plots [corresponding to Figs. 3(a) and 3(b)] provides a simulated switching curve for the momentary peak power in Fig. 3(d) (dotted lines) that fits the measured curve very well. The remaining deviation to the switching curve calculated with a simple $\mathrm{cw} \operatorname{model}^{1,6}$ is caused by the frequency dependence or temporal nonlocality of the cascaded nonlinearity for the 9-ps-long pulses. The fringes in the FW power of the bar arm in the cw switching curve are caused by the generated SH in this arm. ${ }^{6}$ To resolve these details and to decrease the deviation between the momentary-power switching curves from pulsed measurements and a cw switching curve the non-local regime of the cascaded nonlinearity needs to be suppressed better and the nonlinearity needs to act more instantaneous. This could be done in experiments with longer pulse lengths or in experiments with larger phase-mismatch where the nonlinearity for all spectral components is more uniform. Summarizing the measurement shows that the quadratic cascaded nonlinearity in $\mathrm{LiNbO}_{3}$ waveguides with a wave-vector mismatch larger than $\Delta k L>16 \pi$ can be considered quasiinstantaneous down to pulse lengths of $\approx 10 \mathrm{ps}$.

In conclusion, it was shown both experimentally and numerically that quadratic nonlinear waveguide couplers are well understood, thus quantitatively predicting the resulting pulse break-up, asymmetry, and back switching. The obtained detailed knowledge can be used to predictably engineer nonlinear optical waveguide systems based on the $\mathrm{LiNbO}_{3}$ platform, as well as to provide physical understanding of the process of light localization in quadratic nonlinear waveguide arrays. ${ }^{14}$

We acknowledge the support by the Australian Research Council Centre of Excellence program (project 
CE110001018), the Australian Academy of Science, and the International Bureau of the Federal Ministry of Education and Research (BMBF), Germany (Australia-Germany Researcher Mobility Call 2010-2011). We thank Professor Dr. W. Sohler and R. Ricken from the University of Paderborn for the waveguide fabrication.

${ }^{1}$ S. M. Jensen, IEEE J. Quantum Electron. QE-18, 1580 (1982).

${ }^{2}$ P. Li Kam Wa, J. E. Sitch, N. J. Mason, J. S. Roberts, and P. N. Robson, Electron. Lett. 21, 26 (1985).

${ }^{3}$ A. A. Maier, Y. N. Serdyuchenko, K. Y. Sitarskii, M. Y. Shchelev, and I. A. Shcherbakov, Sov. J. Quantum Electron. 17, 735 (1987).

${ }^{4}$ S. R. Friberg, Y. Silberberg, M. K. Oliver, M. J. Andrejco, M. A. Saifi, and P. W. Smith, Appl. Phys. Lett. 51, 1135 (1987).
${ }^{5}$ K. Al-hemyari, A. Villeneuve, J. U. Kang, J. S. Aitchison, C. N. Ironside, and G. I. Stegeman, Appl. Phys. Lett. 63, 3562 (1993).

${ }^{6}$ R. Schiek, Opt. Quantum Electron. 26, 415 (1994); erratum 27, 133 (1995).

${ }^{7}$ R. Schiek, Y. Baek, G. Krijnen, G. I. Stegeman, I. Baumann, and W. Sohler, Opt. Lett. 21, 940 (1996).

${ }^{8}$ R. Schiek, L. Friedrich, H. Fang, G. I. Stegeman, K. R. Parameswaran, M.-H. Chou, and M. M. Fejer, Opt. Lett. 24, 1617 (1999).

${ }^{9}$ M. Romagnoli, S. Trillo, and S. Wabnitz, Opt. Quantum Electron. 24, S1237 (1992).

${ }^{10}$ B. K. Nayar, N. Finlayson, N. J. Doran, S. T. Davey, D. L. Williams, and J. W. Arkwright, Opt. Lett. 16, 408 (1991).

${ }^{11}$ R. Schiek and T. Pertsch, Opt. Mater. Express 2, 126 (2012).

${ }^{12}$ R. Schiek, Y. Baek, and G. I. Stegeman, J. Opt. Soc. Am. B 15, 2255 (1998).

${ }^{13}$ M. Bache, O. Bang, J. Moses, and F. W. Wise, Opt. Lett. 32, 2490 (2007).

${ }^{14}$ R. Iwanow, R. Schiek, G. I. Stegeman, T. Pertsch, F. Lederer, Y. Min, and W. Sohler, Phys. Rev. Lett. 93, 113902 (2004). 